OPEN ACCESS

Edited by:

John T. Fisher,

Queen's University, Canada

Reviewed by:

William Sheel,

The University of British Columbia,

Canada

Gerrard Rafferty,

King's College London,

United Kingdom

*Correspondence:

Guilherme Fregonezi

fregonezi.guilherme@gmail.com

Specialty section:

This article was submitted to

Respiratory Physiology,

a section of the journal

Frontiers in Physiology

Received: 21 January 2019

Accepted: 18 July 2019

Published: 02 August 2019

Citation:

da Fonsêca JDM, Resqueti VR, Benício K, Fregonezi G and Aliverti A

(2019) Acute Effects of Inspiratory

Loads and Interfaces on Breathing

Pattern and Activity of Respiratory

Muscles in Healthy Subjects.

Front. Physiol. 10:993.

doi: 10.3389/fphys.2019.00993

\section{Acute Effects of Inspiratory Loads and Interfaces on Breathing Pattern and Activity of Respiratory Muscles in Healthy Subjects}

\begin{abstract}
Jéssica Danielle Medeiros da Fonsêca ${ }^{1,2}$, Vanessa Regiane Resqueti ${ }^{1,2}, K_{\text {Kadja Benício }}^{1,2}$, Guilherme Fregonezi ${ }^{1,2 *}$ and Andrea Aliverti ${ }^{3}$

' PneumoCardioVascular Lab/HUOL, Hospital Universitário Onofre Lopes, Empresa Brasileira de Serviços Hospitalares and Departamento de Fisioterapia Universidade Federal do Rio Grande do Norte, Natal, Brazil, ${ }^{2}$ Laboratório de Inovação Tecnológica em Reabilitação, Departamento de Fisioterapia, Universidade Federal do Rio Grande do Norte, Natal, Brazil, ${ }^{3}$ Dipartimento di Elettronica, Informazione e Bioingegneria, Politecnico di Milano, Milan, Italy
\end{abstract}

Objectives: The aim of this study was to evaluate the acute effects of different inspiratory loads and different interfaces on the breathing pattern and activity of the respiratory muscles.

Methods: Twenty healthy adults were recruited and assigned to two groups (20 and $40 \%$ of the Maximal Inspiratory Pressure) by way of randomized crossover allocation. Subjects were evaluated during quiet breathing, breathing against inspiratory load, and recovery. The measurements were repeated using two different interfaces (nasal and oral). Chest wall volumes and respiratory muscle activity were assessed with optoelectronic plethysmography and surface electromyography, respectively.

Results: During the application of inspiratory load, significant changes were observed in the respiratory rate $(p<0.04)$, inspiratory time $(p<0.02)$, minute ventilation $(p<0.04)$, tidal volume $(p<0.01)$, end-inspiratory volume $(p<0.04)$, end-expiratory volume $(p<0.03)$, and in the activity of the scalene, sternocleiomastoid, and parasternal portion of the intercostal muscles (RMS values, $p<0.01$ ) when compared to quiet breathing, regardless of the load level or the interface applied. Inspiratory load application yielded significant differences between using nasal and oral interfaces with an increase in the tidal volume $(p<0.01)$, end-inspiratory volume $(p<0.01)$, and electrical activity of the scalene and sternocleiomastoid muscles $(p<0.01)$ seen with using the nasal interface.

Conclusion: The addition of an inspiratory load has a significant effect on the breathing pattern and respiratory muscle electrical activity, and the effects are greater when the nasal interface is applied.

Keywords: respiratory muscles, healthy subjects, electromyography, plethysmography, physiology

\section{INTRODUCTION}

The application of an inspiratory load is a method used for inspiratory muscle training (IMT) (Hostettler et al., 2011); it helps to increase endurance and respiratory muscle strength in order to improve lung function (Sasaki, 2007), functional capacity (Shakouri et al., 2015), and efficacy in bronchial airway clearance (Oliveira et al., 2009). Three IMT techniques reported in literature 
are more commonly used: voluntary isocapnic hyperpnea, flow resistive loading, and pressure threshold loading (McConnell and Romer, 2004).

In inspiratory flow resistance devices, subjects perform inspirations through an orifice of variable diameter; the smaller the orifice diameter, the greater the resistive inspiratory load. However, these devices depend on the inspiratory flow generated by the subject. The new generation of electronic devices have overcome this limitation, in that, they are based on an electronically controlled variable flow resistive load. It has been reported that its use produces higher training loads and better inspiratory function when compared to pressure threshold devices (Langer et al., 2015). However, few studies have evaluated the compensatory mechanisms employed by the respiratory system when breathing is done against electronically controlled variable flow resistive loads.

The act of breathing is a vital biological process that, in normal conditions, occurs through the nose, for the purpose of filtering, humidifying, and heating the inspired air. Warmed, humidified air minimizes airway constriction and the resultant increased airway resistance. In addition, it improves upper airway mucociliary function, which augments the elimination of secretions. Despite this, the respiratory resistance devices available in the market only use mouthpieces (oral airway). The use of a nasal interface, for training inspiratory muscles, could be more favorable physiologically and more viable for individuals who are unable to hold a mouthpiece, such as patients with facial trauma or neurological problems that cause weakness of the facial muscles. Held et al. (2008) performed a protocol of muscular training and nasal breathing in mouth breathing children (MBS) and reported improvements in respiratory muscle strength and nasal respiratory flow. This study indicates that applied inspiratory loads using a nasal interface could induce compensatory mechanisms in the activities of respiratory muscles, resulting in an improved breathing pattern. However, the mechanism that provides these advantages when using a nasal interface is not well-understood.

Therefore, considering the possible benefits of nasal breathing on the respiratory system, we hypothesized that the use of a nasal interface will improve breathing pattern and the variation of chest wall volumes, as well as the electrical activity of the respiratory muscles.

\section{MATERIALS AND METHODS}

\section{Type of Study and Subjects}

This research was carried out at the PneumoCardioVascular Lab, in the Federal University of Rio Grande do Norte/Brazilian Company of Hospital Services (UFRN/EBSERH) in the city of Natal/RN. We included, in the study, young adults belonging to the male and female sexes, presenting normal values of pulmonary function, with ages ranging from 18 to 30 years and with Body Mass Indexes (BMIs) between 18 and $29.9 \mathrm{~kg} / \mathrm{m}^{2}$. We excluded individuals that failed to perform the tests/protocol, as well as those who presented irregularities during data analysis or voluntarily requested their removal from the study.
This study was approved by the Hospital Research Ethics Committee (number 428.987). The participants signed the consent form, which was drafted according to the tenets of the Helsinki declaration related to research on human beings, including special attention to confidentiality, respect for the human person, beneficence, autonomy, and non-maleficence.

\section{Pulmonary Function}

Spirometry was performed using the KoKo DigiDoser ${ }^{\circledR}$ spirometer (Longmont, CO, United States). The evaluations were performed according to the criteria of acceptability and reproducibility of the American Thoracic Society/European Respiratory Society (ATS/ERS) (American Thoracic Society/European Respiratory Society, 2002) and the reference values were derived from the predicted values for Brazilian adults (Pereira et al., 2007).

Respiratory muscle strength was determined by measuring maximal inspiratory pressure (MIP), maximal expiratory pressure (MEP), and sniff nasal inspiratory pressure (SNIP) using a digital manovacuometer (NEPEB-LabCare/UFMG, Belo Horizonte, Brazil). The evaluations were performed according to American Thoracic Society/European Respiratory Society (2002) acceptability and reproducibility criteria. Reference values previously published by Neder et al. (1999) were used for MIP and MEP, whereas the reference values of Araújo et al. (2012) were used for the SNIP. For all pulmonary function variables, the absolute values and percentage-predicted values were used for analysis.

\section{Optoelectronic Plethysmography}

The measurement of the volumes of the chest wall $(\mathrm{CW})$ and its compartments: the pulmonary rib cage $\left(\mathrm{R}_{C p}\right)$, the abdominal rib cage $\left(\mathrm{R}_{\mathrm{Ca}}\right)$, and the abdomen $(\mathrm{Ab})$ was performed by Optoelectronic Plethysmography (OEP, BTS ${ }^{\circledR}$, Milan, Italy), in which volumes were obtained following an experimental model according to the Gauss theorem (Cala et al., 1996). Before each data acquisition, the equipment was calibrated at a frequency of $60 \mathrm{~Hz}$, the maximal frequency to achieve a quality signal for detecting chest wall movement. Six cameras positioned around the subject (three in the anterior region and three in the posterior region) captured the movement variation of 89 reflective markers. They were fixed at specific points in the anterior, posterior, and lateral regions of the thorax, between the clavicles and the anterior superior iliac spine, distributed in seven horizontal lines and in five pre-defined vertical columns, beside the addition of extra points. This setting was used to improve accuracy in volume assessment and to anatomically define the three regions or compartments of the chest wall, in which the frontiers between $\mathrm{R}_{C p}$ and $\mathrm{R}_{C a}$ is at the level of the xiphoid appendix and between $\mathrm{R}_{C a}$ and $\mathrm{Ab}$, along the costal margin anteriorly, and at the lowest point of the costal inferior margin posteriorly (Aliverti and Pedotti, 2003).

From the OEP data, the following variables were analyzed for the CW and its compartments: tidal volume [tidal volume in chest wall $\left(\mathrm{V}_{T, C W}\right)$, tidal volume in pulmonary rib cage $\left(\mathrm{V}_{T, R C p}\right)$, tidal volume in abdominal rib cage $\left(\mathrm{V}_{T, R C a}\right)$, and tidal volume in abdomen $\left.\left(\mathrm{V}_{T, A b}\right)\right]$; end-inspiratory volumes 
[end-inspiratory volume in chest wall $\left(\mathrm{EIV}_{\mathrm{CW}}\right)$, end-inspiratory volume in pulmonary rib cage $\left(\mathrm{EIV}_{R C p}\right)$, end-inspiratory volume in abdominal rib cage $\left(\mathrm{EIV}_{R C a}\right)$, and end-inspiratory volume in abdomen $\left(\mathrm{EIV}_{A b}\right)$ ]; end-expiratory volumes [endexpiratory volume in chest wall $\left(E E V_{C W}\right)$, end-expiratory volume in pulmonary rib cage $\left(\mathrm{EEV}_{R C p}\right)$, end-expiratory volume in abdominal rib cage $\left(\mathrm{EEV}_{R C a}\right)$, and end-expiratory volume in abdomen $\left.\left(\mathrm{EEV}_{A b}\right)\right]$; respiratory rate $(\mathrm{RR})$; inspiratory time $\left(\mathrm{T}_{I}\right)$; expiratory time $\left(\mathrm{T}_{E}\right)$; inspiratory flow $\left(\right.$ Flow $\left._{I}\right)$; expiratory flow $\left(\right.$ Flow $\left._{E}\right)$; minute volume $(\mathrm{MV})$; and total time of respiratory cycle $\left(\mathrm{T}_{T O T}\right)$.

\section{Surface Electromyography (sEMG)}

The surface electromyography (sEMG) was performed following the recommendations of the International Society of Electrophysiology Kinesiology (ISEK) (Merletti et al., 1999). Myoelectric signals were recorded using the electromyographic TeleMyo DTS Desk Receiver ${ }^{\circledR}$ (Noraxon USA Inc., Scottsdale, AZ, United States) and four wireless sensors Clinical DTS (Noraxon USA Inc., Scottsdale, AZ, United States) with a 20-500 $\mathrm{Hz}$ pass filter-band, 1000 gain, 16-bit resolution, and a common mode rejection rate greater than $120 \mathrm{~dB}$. Bipolar double trace Ag/AgCl (Miotec, Porto Alegre, Brazil) passive surface self-adhesive electrodes were placed on these muscles: scalene (SCL) at a distance of $5 \mathrm{~cm}$ from the sternumclavicular joint and $2 \mathrm{~cm}$ above this point (Cunha et al., 2005), sternocleiomastoid (SCM) in the lower third of the distance between the mastoid process and the sternum-clavicular joint (Falla et al., 2002), abdominal rectus (RA) at $4 \mathrm{~cm}$ from the umbilical scar, and in the parasternal portion of the intercostal muscle (IC) over the second intercostal space and $3 \mathrm{~cm}$ from the sternum (Maarsingh et al., 2000), and all placed on the right side of the body to minimize cardiac noise interference. Before placing the electrodes, the skin region was prepared using an abrasive gel to reduce impedance to capturing the electrical signal. The software used to capture, process, and store the signals was MR 3.2 (Noraxon, Inc., Scottsdale, AZ, United States). Raw data was analyzed by means of RMS (root mean square) and standardized from respiratory baseline values (Soderberg and Knutson, 2000).

\section{Inspiratory Load}

The effect of inspiratory loads with different interfaces was evaluated with an electronic variable resistive load device (POWERBreathe ${ }^{\circledR}$ KH5, International, Ltd., Warwickshire, United Kingdom). The POWERBreathe ${ }^{\circledR} \mathrm{KH} 5$ is an electronically controlled, variable flow resistance device generally used for IMT, in which an absolute initial load is assigned and successively reduced depending on the inspiratory flow generated by the subject.

\section{Randomization and Study Design}

The participants were categorized into two groups in randomized crossover design $\left(\mathrm{MIP}_{20 \%}\right.$ and $\left.\mathrm{MIP}_{40 \%}\right)$. By way of a simple draw of an opaque envelope, they were grouped according to the initial resistive load applied (20 and 40\% of MIP, respectively). Assessments were carried out in two stages: (1) clinical, spirometric, and respiratory muscle strength; and (2) chest wall volumes concomitant to the activity of respiratory muscles. During stage 2, the assessment consisted of three steps of 30 s each: (1) spontaneous quiet breathing (QB), (2) breathing against inspiratory load (Load), and (3) recovery (Rec) (Figure 1).

Due to the volume of the EMG signal data, with acquisitions at $1500 \mathrm{~Hz}$ and Optoelectronic Plethysmography at $60 \mathrm{~Hz}$, the total duration for each step was limited to 30 s so the signals could be registered in a synchronized mode.

Two load intensities (20 and 40\% of MIP) were applied and used with two different interfaces- oral and nasal, the order of which was designated by the same randomization process. The POWERBreathe was the resistive valve used for both interfaces. For the nasal interface, a patented device [PI 0164278 - INPI Brazil], which consists of an orofacial mask associated with the resistive valve, was used. During its use, participants were asked to breath exclusively through the nose.

\section{Data Processing}

A routine was developed in MatLab (MathWorks, Inc., Natick, MA, United States) to synchronize sEMG and OEP data. The resulting file was used to analyze the breathing pattern data, breath-to-breath data, on Diamov (a customized software designed and developed at the Politécnico di Milano, Italy). Furthermore, the file was also used to select the length of time and calculate RMS on MR 3.2 software.

The digital filters applied to EMG signals were electrocardiogram filter for cardiac electric signal removal, rectification, and smoothing filter for the elimination of non-reproducible signals (50 ms window).

\section{Sample Size and Statistical Analysis}

Sample size was established considering the tidal volume as the main variable. Five subjects were evaluated using hypothetical one-way ANOVA during all three steps (QB, Load, and Recovery). A sample size ranging between 15 to 21 subjects for each group with a mean of 19 subjects was estimated using the following: an alpha error of 0.05 with bilateral distribution, and a test power of $80 \%$, an effect size resulting from the ANOVA test described through the partial eta squared $\left(\eta_{\mathrm{p}}^{2}\right)$ values for the groups $\left(\mathrm{MIP}_{20 \%}\right.$ with an oral interface $\left({ }^{\eta_{\mathrm{p}}^{2}}=0.36\right), \mathrm{MIP}_{20 \%}$ with a nasal interface $\left(\eta_{\mathrm{p}}^{2}=0.38\right), \mathrm{MIP}_{40 \%}$ with an oral interface $\left(\eta_{\mathrm{p}}^{2}=0.42\right)$, and $\mathrm{MIP}_{40 \%}$ with a nasal interface $\left(\eta_{\mathrm{p}}^{2}=0.52\right)$.

Data normality was verified using the Shapiro-Wilk test. The Friedman test was used to analyze the steps of spontaneous quiet breathing, breathing against inspiratory load, and recovery, and in the case of a significant difference, Dunn's post hoc test was applied. Comparisons between the oral and nasal interfaces, and that between 20 and $40 \%$ of the MIP loads were performed using the Wilcoxon test. For data analysis, the GraphPad Prism 6.0 program (GraphPad Software, San Diego, CA, United States) for Windows was used. The power $(\beta)$ and effect size (ES) were estimated and are detailed in the results section of this study. For all statistical analyses, a level of significance, $p<0.05$ with bilateral distribution was adopted. 


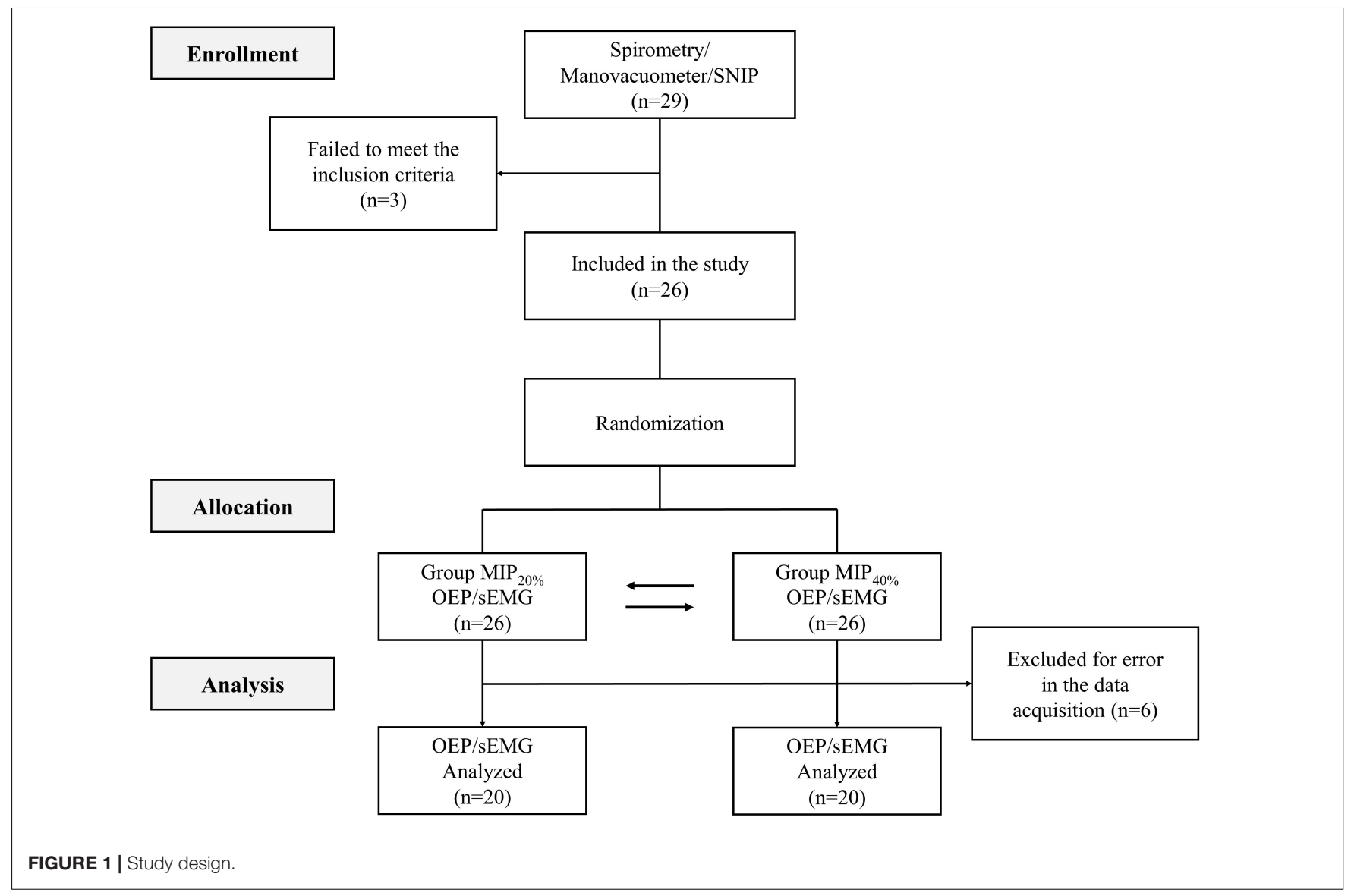

The sample size calculation, $\beta$, and ES of the study were calculated using GPower software version 3.1.9.2 (University of Düsseldorf, Kiel, Germany).

\section{RESULTS}

Twenty-nine subjects were screened, of which three did not meet the inclusion criteria. Six subjects were excluded due to low quality of their electromyography signals and/or OEP volumes, resulting in a final sample of 20 subjects. The sample description and characterization referring to baseline anthropometric and pulmonary function data are shown in Table 1.

\section{Chest Wall and Compartmental Volumes}

Figure 2 shows total chest wall and compartmental tidal volumes $\left(\mathrm{V}_{T, C W}, \mathrm{~V}_{T, R C p}, \mathrm{~V}_{T, R C a}\right.$, and $\left.\mathrm{V}_{T, A b}\right)$, while operational volume variations (end-inspiratory and end-expiratory total and compartmental volumes) are shown in Figure 3.

Regardless of the imposed load, significant increases in $\mathrm{V}_{T, C W}$ were observed during the Load step compared with the QB and Rec steps $(p<0.01)$. $\mathrm{V}_{T, C W}$ and compartmental volumes were higher when using the nasal interface $(p<0.05)$ compared with the volumes observed when the oral interface was used. The $\mathrm{MIP}_{40 \%}$ group presented an increase of 0.16 and $0.15 \mathrm{~L}$ with the oral and nasal interfaces, respectively, than the $\mathrm{MIP}_{20} \%$ group. However, these values were not statistically significant.

Regarding the variations of operational volumes during breathing against inspiratory load, an increase in $\mathrm{EIV}_{C W}$ $\left(\mathrm{MIP}_{20 \%}\right.$ : oral and nasal interfaces, $p<0.01 ; \mathrm{MIP}_{40 \%}$ : oral interface, $p=0.04$ and nasal interface, $p<0.01$ ) and a reduction in $\mathrm{EEV}_{C W}\left(\mathrm{MIP}_{20 \%}\right.$ : oral interface, $p=0.02$ and nasal interface, $p=0.01 ; \mathrm{MIP}_{40 \%}$ : oral interface, $p=0.03$ and nasal interface, $p=0.01)$ resulted.

During the Load step, there was a mean increase of $0.38 \mathrm{~L}$ in $\operatorname{EIV}_{R C p}(p<0.01$, regardless of the load intensity and interface used) and a mean increase of $0.22 \mathrm{~L}$ in $\operatorname{EIV}_{R C a}\left(\mathrm{MIP}_{20 \%}\right.$ : oral and nasal interface, $p<0.01 ; \mathrm{MIP}_{40 \%}$ : oral interface, $p=0.02$ and nasal interface, $p<0.01$ ), when compared to the $\mathrm{QB}$ and $\operatorname{Rec}$ steps, resulting in a greater $\mathrm{EIV}_{C W}$. There was no significant variation in the abdomen. The $\mathrm{EEV}_{C W}$ reduction, in response to an increased ventilatory demand, was mostly related to the volume generated in the abdominal compartment $\left(\mathrm{EEV}_{A b}\right)$ $\left(\mathrm{MIP}_{20 \%}\right.$ : oral interface, $p=0.03$ and nasal interface, $p=0.01$; $\mathrm{MIP}_{40 \%}$ : oral and nasal interface, $p=0.01$ ). The abdominal compartment had a mean volume decrease of $0.16 \mathrm{~L}$, whereas the rib cage maintained a constant volume.

Comparing the Load step when using the nasal and oral interfaces, EIV $\mathrm{EW}_{\mathrm{W}}$ was higher with loads imposed via the nasal airway $\left(\mathrm{MIP}_{20 \%}\right.$ and $\left.\mathrm{MIP}_{40 \%}: p<0.01\right)$, with no differences in relation to $\mathrm{EEV}_{C W}$. 
TABLE 1 | Sample description.

\begin{tabular}{|c|c|}
\hline Description & \\
\hline Subjects (n) & 20 \\
\hline Gender F/M & $11 / 9$ \\
\hline Age, yrs & $24.4 \pm 2.72$ \\
\hline BMI $\left(\mathrm{kg} / \mathrm{m}^{2}\right)$ & $23.9 \pm 2.7$ \\
\hline \multicolumn{2}{|c|}{ Pulmonary function } \\
\hline $\mathrm{FEV}_{1} \mathrm{~L}$ & $3.59 \pm 0.55$ \\
\hline$\%$ predicted & $95 \pm 9$ \\
\hline FVC L & $4.29 \pm 0.75$ \\
\hline$\%$ predicted & $95 \pm 10$ \\
\hline FEV1/FVC & $0.84 \pm 0.07$ \\
\hline$\%$ predicted & $100 \pm 8$ \\
\hline $\mathrm{MIP} \mathrm{cmH}_{2} \mathrm{O}$ & $116.2 \pm 25.21$ \\
\hline$\%$ predicted & $104 \pm 24$ \\
\hline MEP $\mathrm{cmH}_{2} \mathrm{O}$ & $118 \pm 26.29$ \\
\hline$\%$ predicted & $98 \pm 10$ \\
\hline $\mathrm{SNIP} \mathrm{cmH}_{2} \mathrm{O}$ & $104.8 \pm 19.4$ \\
\hline$\%$ predicted & $97 \pm 21$ \\
\hline \multicolumn{2}{|c|}{$\begin{array}{l}\text { Values are mean } \pm S D \text {. F, female; M, male; yrs, years; BMI, Body Mass Index; } \\
\text { kg, kilograms; } m \text {, meters; FEV } 1 \text {, Forced Expiratory Volume in the first second; L, } \\
\text { liters; FVC, Forced Vital Capability; MIP, Maximum Inspiratory Pressure; } \mathrm{CmH}_{2} \mathrm{O} \text {, } \\
\text { centimeters of water; MEP, Maximum Expiratory Pressure; SNIP, Sniff Nasal } \\
\text { Inspiratory Pressure. }\end{array}$} \\
\hline
\end{tabular}

As shown in Table 2, $\mathrm{T}_{T O T}, \mathrm{~T}_{I}, \mathrm{MV}$, Flow ${ }_{I}$, Flow , and $\mathrm{RR}$ varied during breathing against both inspiratory loads $\left(\mathrm{MIP}_{20 \%}\right.$ and $\left.\mathrm{MIP}_{40 \%}\right)$ compared to $\mathrm{QB}$, with only $\mathrm{T}_{\text {TOT }}$ not presenting significant variations with the $\mathrm{MIP}_{40 \%}$ load. $\mathrm{T}_{E}$ did not vary in any of the conditions. The comparison between different interfaces and load intensities did not show any statistically significant difference.

\section{Electrical Activity of Respiratory Muscles}

Surface electromyography signals normalized by expressing RMS as percentage of resting conditions are shown in Figure 4. During breathing against both inspiratory loads, RMS of the SCM, SCL, and IC muscles increased $(p<0.01)$ in relation to the $\mathrm{QB}$ and $\mathrm{Rec}$ steps. The comparison between interfaces revealed significantly higher RMS values of the nasal interface for SCM $\left(\mathrm{MIP}_{20 \%}, p<0.01\right.$ and $\mathrm{MIP}_{40 \%}$, $p=0.01)$ and SCL $(p<0.01)$. sEMG signals were higher in $\mathrm{MIP}_{40 \%}$ than $\mathrm{MIP}_{20 \%}$ for SCM $(p<0.01)$, SCL $(p<0.01)$, and IC $(p<0.01)$. The RA muscle did not show any significant variations in its activity when different loads or interfaces were used.

\section{Power and Effect Size}

Table 3 summarizes the effect size and power test for chest wall volumes and the sEMG RMS values of the SCM, SCL, and IC muscles during quiet breathing, load, and recovery steps. It also shows the comparison between the nasal and oral interfaces during the application of inspiratory load.

\section{DISCUSSION}

We studied the acute effects of adding variable flow inspiratory loads using nasal and oral interfaces on the respiratory pattern variations and respiratory muscle activation. The addition of inspiratory loads resulted in: (1) increased tidal volume due to changes in the end-inspiratory volume and end-expiratory volume, the former occurring due to its increase in the $\mathrm{R}_{C p}$ and $\mathrm{R}_{\mathrm{Ca}}$ compartments, and the latter by its decrease in the abdomen compartment; (2) these changes were followed by increased electrical activity of the SCL, SCM and IC muscles; (3) these effects were more evident when using the nasal interface.

This study is a precursor to investigating the effects of inspiratory loads imposed on the nasal airway. We found that the nasal interface (nasal airway) promoted greater lung volume generation and inspiratory muscle activation when compared to the oral interface (oral airway). There are mechanical differences between the nasal and oral airways, depending on the region. The nasal airway has a higher resistance $\left(\mathrm{RN}=0.68 \mathrm{cmH}_{2} \mathrm{O}\right.$ $\mathrm{L}^{-1} \mathrm{~s}^{-1}$ at $0.5 \mathrm{~L} \mathrm{~s}^{-1}$ ) and is relatively fixed, regulated by both the alar muscles and the nasal mucosa. In contrast, the oral airway presents a lower resistance $\left(\mathrm{RO}=0.51 \mathrm{cmH}_{2} \mathrm{O} \mathrm{L}^{-1} \mathrm{~s}^{-1}\right.$ at $0.5 \mathrm{~L} \mathrm{~s}^{-1}$ ); however, there is a high variability in resistance in response to mouth opening (Schiratzki, 2009; Strohl et al., 2012). A possible explanation for our results could be attributed to respiratory tract physiology, since the resistance resulting from nasal airway diameter is higher than that from the oral airway. This would increase the energy needed to generate inspiratory cycles, thus requiring a greater muscle recruitment to promote volume generation.

Thus, we can conclude that the effects of using the nasal interface were potentially better for the respiratory system. These results are aligned with those presented by Held et al. (2008). They demonstrated that a protocol of respiratory muscle training and nasal breathing performed in MBS children improved respiratory muscle strength and nasal inspiratory flow. These authors also reported the importance of nasal respiratory training for the reestablishment of lung volume and nostril elasticity in the studied subjects. Barbiero et al. (2007) observed improvements in forced vital capacity, Tiffeneau score, respiratory muscle strength, and daily habits in mouth breathers after therapy with re-expansive respiratory exercises associated with respiratory biofeedback. However, both studies used simplistic methodologies to evaluate the repercussions on the respiratory system and did not discuss the effects on breathing pattern or respiratory muscle activity.

The increase in chest wall $\left(\mathrm{V}_{T, C W}\right)$ and compartmental $\left(\mathrm{V}_{T, R C P}, \mathrm{~V}_{T, R C a}\right.$, and $\left.\mathrm{V}_{T, A b}\right)$ volumes was similar in both groups and although these volumes presented higher values in $\mathrm{MIP}_{40 \%}$ the change was not significant. Da Gama et al. (2013) analyzed the acute effects of increased inspiratory loads in healthy subjects using sEMG and OEP. Their results showed differences in volume generation depending on gender. It showed that for women, $20 \%$ of MIP is sufficient to generate significant changes, whereas for men, this change is only observed on using loads higher than $30 \%$. Nobre et al. (2007) analyzed pulmonary ventilation using scintigraphy during inspiratory muscle endurance tests with 10 , 

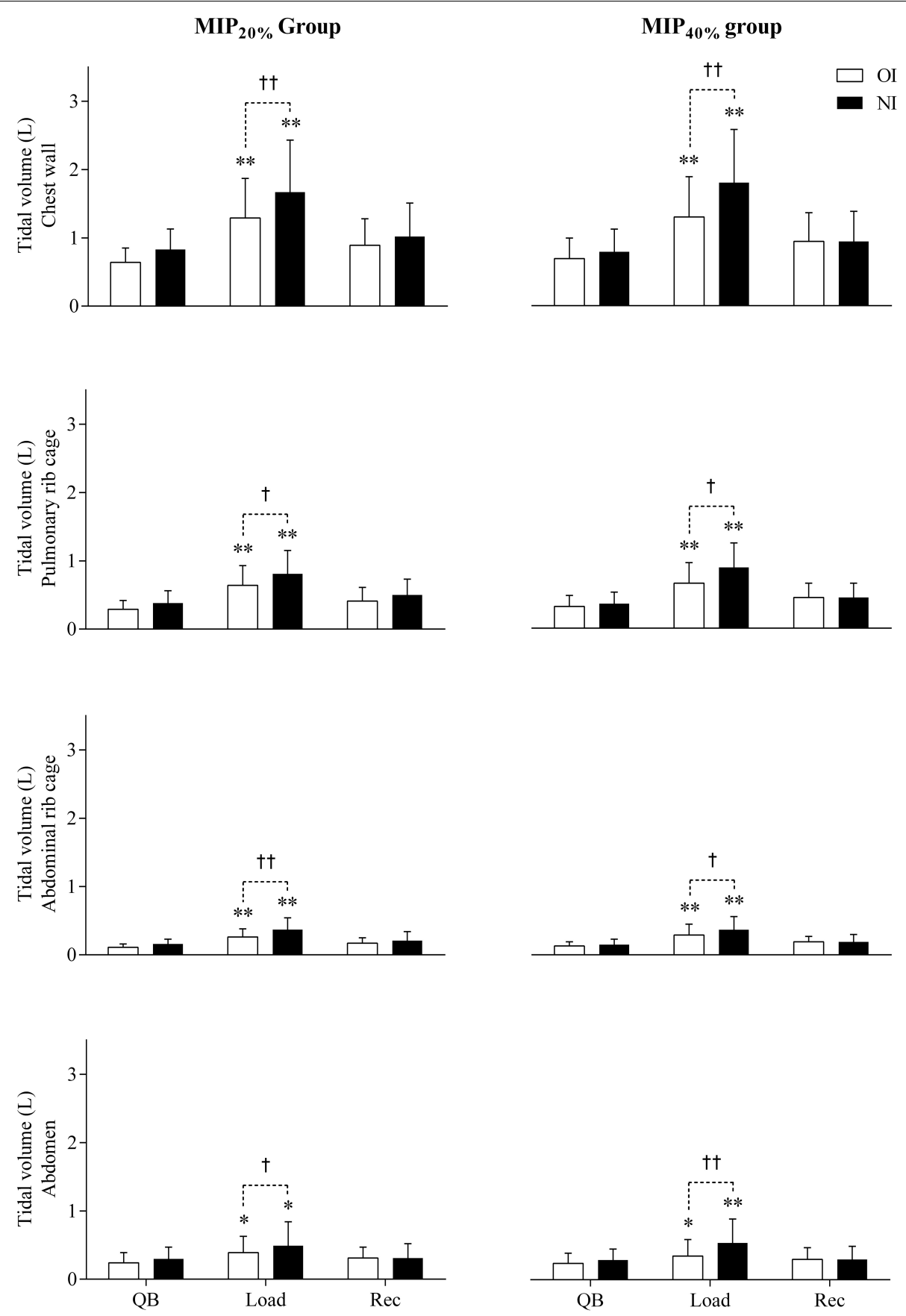

FIGURE 2 | Variations of chest wall and its compartments tidal volume. QB, quiet spontaneous breathing; Load, breathing against inspiratory load; Rec, recovery; Ol, oral interface; $\mathrm{NI}$, nasal interface; L, liters. * $p<0.05,{ }^{* *} p<0.01$ for Friedman test with post hoc Dunne's comparison with quiet spontaneous breathing. $\dagger p<0.05$, $\uparrow \uparrow p<0.01$ for Wilcoxon test in the comparison between oral vs. nasal interface.

20 , and $30 \%$ of MIP in healthy women. Their results corroborate our results, suggesting that the use of inspiratory resistances greater than $20 \%$ of MIP was able to promote an increase in the pulmonary volume, and showed no significant differences between 20 and $30 \%$ of MIP as well.

The tidal volume is represented by the differences between end-inspiratory volume and end-expiratory volume generation
(Wilkens et al., 2010). Alterations in respiratory drive occur in response to increased work demand resulting from inspiratory load imposition or physical exercise. In our subjects, both groups presented an increase in $\mathrm{V}_{T, C W}$ in response to an increase in the EIV in the rib cage compartments, due to the recruitment of the rib cage inspiratory muscles (SCM, SCL, and IC). By reducing the $\mathrm{EEV}$, the abdomen compartment also contributes 


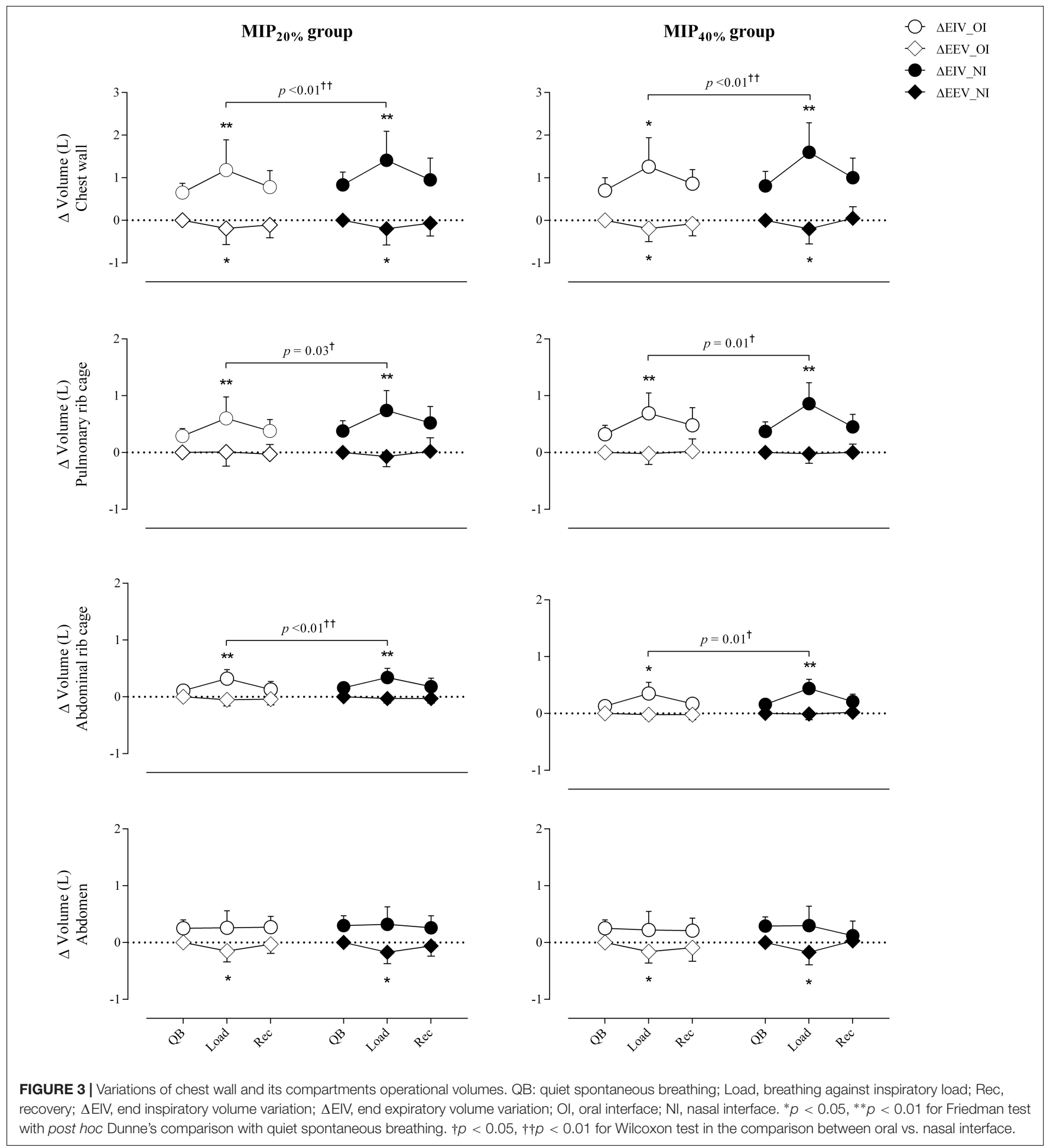

to the increase of the $\mathrm{V}_{T, C W}$. These results were previously described by Aliverti et al. (1997) in a study with healthy adults, which showed an increased EIV $_{C W}$ and decreased $\mathrm{EEV}_{C W}$ in response to ventilation increased. These authors observed a reduction of $0.98 \mathrm{~L}$ in $\mathrm{EEV}_{C W}$ during exercise and identified the abdomen as the volume generator, which corroborates our study. Sanna et al. (1999) also reported changes in chest wall kinematics and respiratory muscles activity in healthy subjects during exercise, and attributed the ventilation increase to the recruitment of $\mathrm{EIV}_{R c p}$ and $\mathrm{EIV}_{\text {Rca }}$, which are in agreement with our results. Both authors observed that in response to an increased ventilatory demand due to exercise, there is an increase 
in $\mathrm{V}_{T}$ of the rib cage associated with an increase in EIV, whereas the increase in $\mathrm{V}_{T, A b}$ results only from a reduced EEV. They also suggested the importance of this arrangement in respiratory mechanics physiology.

The $\mathrm{EEV}_{A b}$ reduction associated with the constant volume in the rib cage $\left(\mathrm{R}_{C p}\right.$ and $\left.\mathrm{R}_{C a}\right)$ is a mechanism that supports the diaphragm muscle action. This increases the diaphragm preinspiratory length and prevents its excessive shortening during inspiration. In contrast, EIV increase associated with the rib cage volume, maintaining constant volume in the abdomen, favors the shortening of the chest wall inspiratory muscles during inspiration, preventing their excessive pre-inspiratory stretching (Aliverti et al., 1997). These authors also showed that EEV increase is a mechanism for optimizing ventilation regardless of exercise modality.

The electromyographic analysis improves the interpretation of our results. We found an increased activity in the SCM, SCL, and IC muscles during breathing against added load when compared to quiet breathing. This shows that the response of the rib cage muscles accompanied the increase in $\mathrm{V}_{T, C W}$ generation, being significantly higher in the SCL and SCM muscles when nasal interface was used, and with the $40 \%$ of MIP.

The chest wall muscles and the diaphragm need to act rhythmically and generate the necessary force needed to maintain ventilation (Han et al., 1993). Some studies evaluated muscle action by measuring the pressures generated by respiratory muscles during exercise (Aliverti et al., 1997; Sanna et al., 1999). They observed a progressive increase in the pressure generated by chest wall muscles during inspiration (Prcm). This was responsible for these muscles shortening, and consequently led to an increase in EIV, causing an expansion of the rib cage. The analysis of the abdominal muscle pressure (Pabm) showed an increase during expiration and reduction during inspiration. The former is related to the increase of EEV in the abdomen, whereas the latter associated with Prcm contributes to the expansion of $\mathrm{R}_{\mathrm{Ca}}$ and an increase of EIV. The abdominal muscles also presented with a third function. Their relaxation during inspiration, associated with Prcm increase, allows the diaphragm to generate greater flow to meet the higher ventilatory demand.

Studies reporting the acute effects of inspiratory loads on the electrical activity of the respiratory muscles in healthy subjects were performed by Da Gama et al. (2013). In contrast to our results, they found a decrease in the activity of the SCM and diaphragm muscles in response to increased inspiratory load above $30 \mathrm{cmH}_{2} \mathrm{O}$. Their results were associated with a possible fatigue in these muscles. However, Nobre et al. (2007) reported similar results to our study, such that an increased activity in the lower rib cage muscles and the SCM were found, the latter not significant due to a higher inspiratory resistance. Walterspacher et al. (2018) evaluated the effect of IMT modalities on the activity of respiratory muscles, concluding that IMT promotes an increase of the SCM, IC, and diaphragm muscles activity. Using a pressure threshold device, Hawkes et al. (2007) reported the acute effects of submaximal inspiratory load on the inspiratory muscle strength of 12 healthy adult subjects. They found variations in the muscle recruitment pattern that was initially promoted by

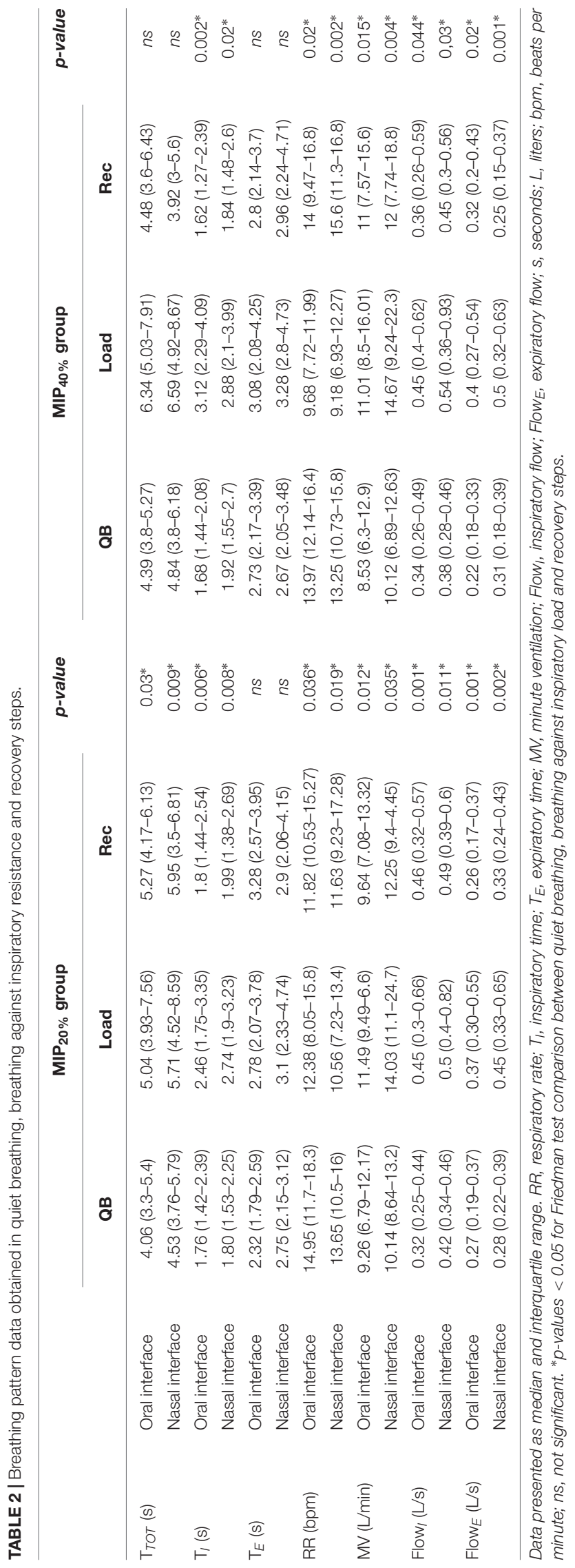




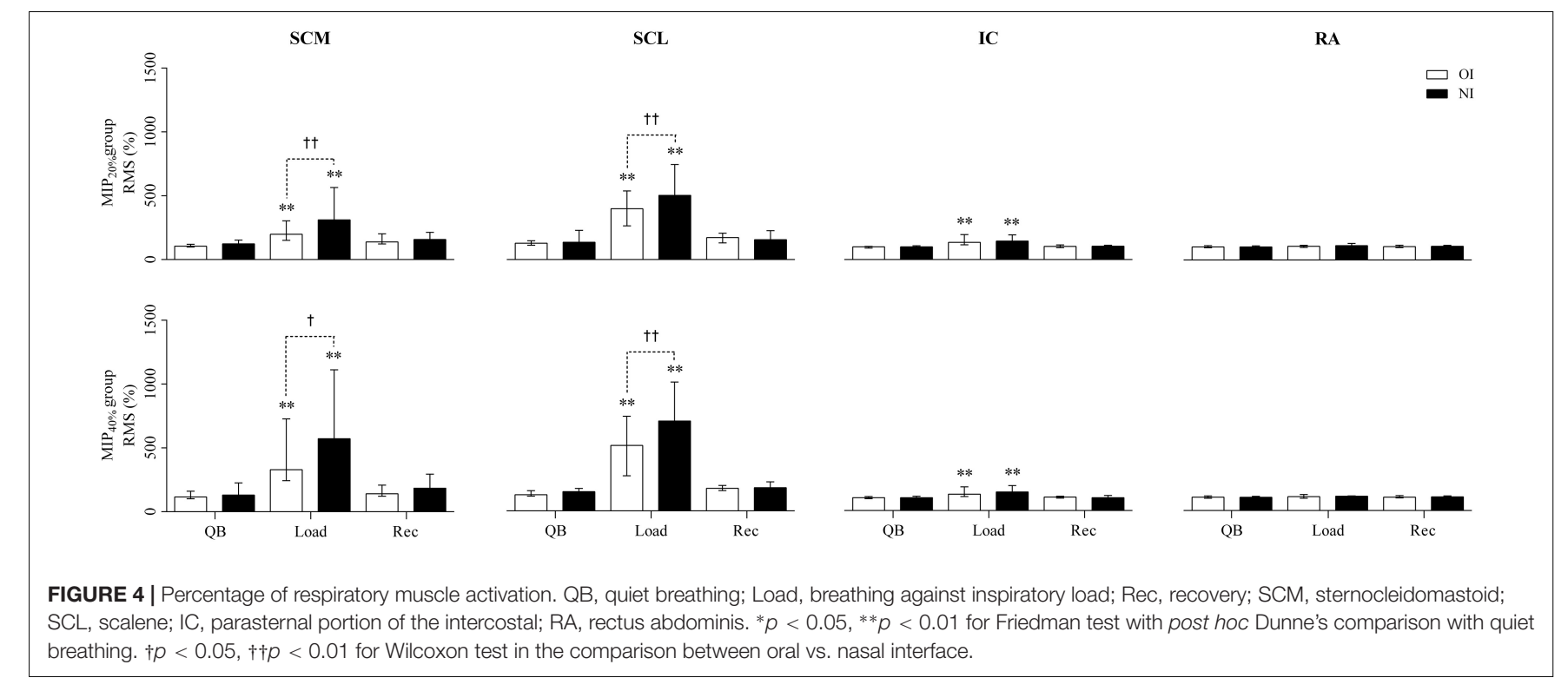

TABLE 3 | Effect size and power test.

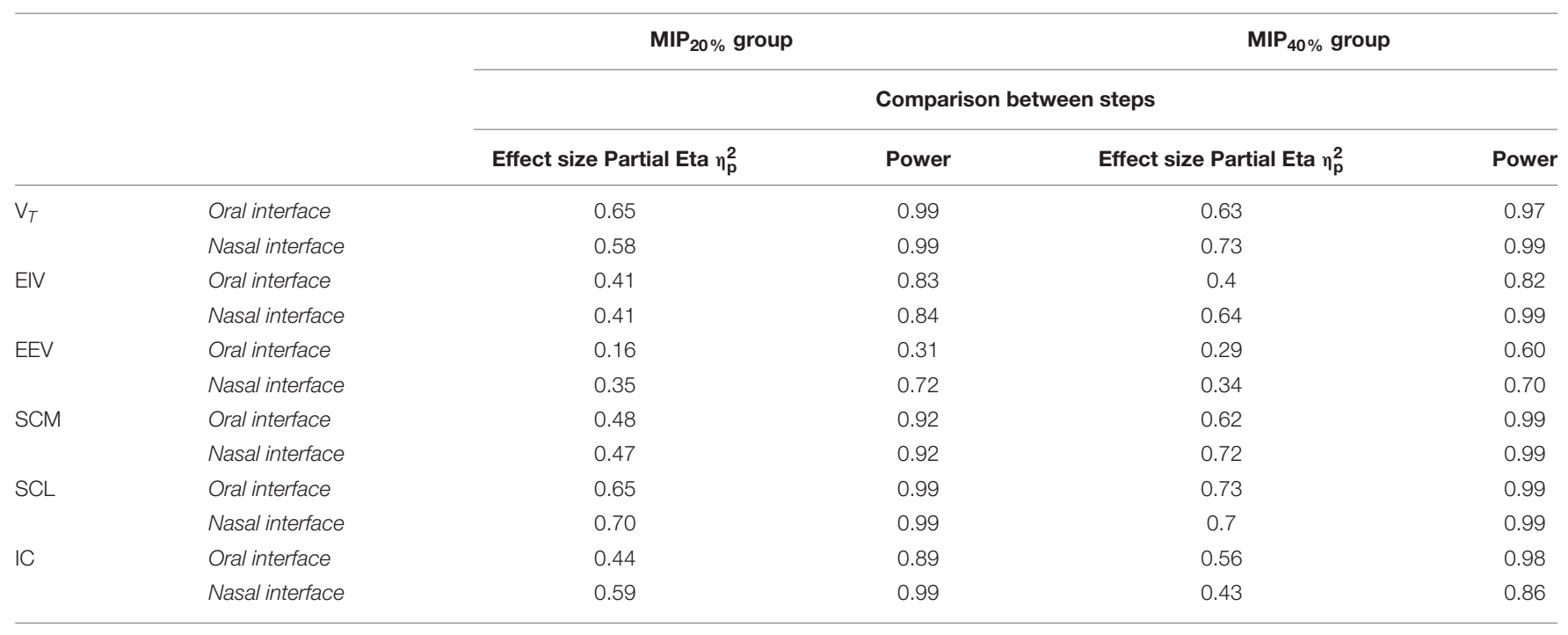

Comparison between oral interface vs. nasal interface during load

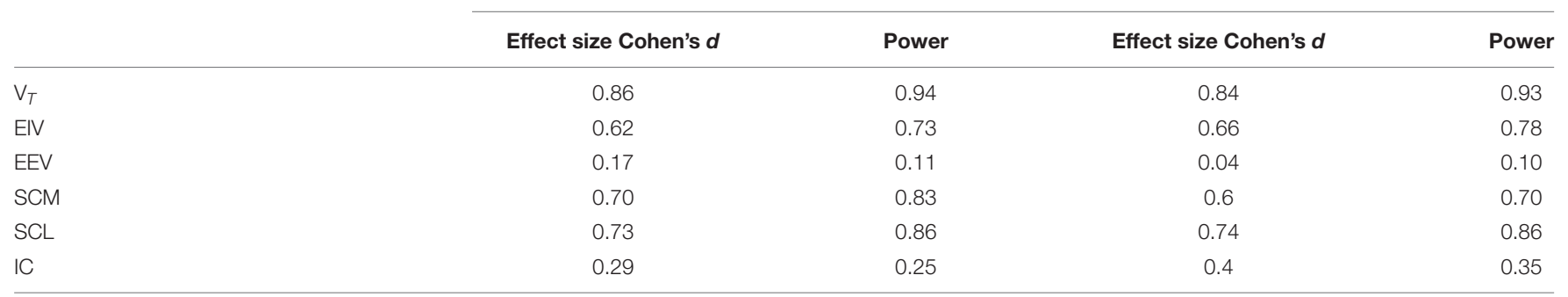

Comparison of chest wall volumes and percentage of respiratory muscle activation intensity between quiet breathing, breathing against inspiratory load and recovery steps and between loading of nasal and oral interfaces. $V_{T}$, tidal volume; EIV, end inspiratory volume; EIV, end expiratory volume; SCM, sternocleidomastoid; SCL, scalene; IC, parasternal portion of the intercostal; Ol, oral interface; NI, nasal interface.

the diaphragm and later assisted by the intercostal muscle, which once activated, showed greater activity than the diaphragm.

Physiologically, the SCL and IC muscles have a primary inspiratory action and greater mechanical advantage (3.4 and
$2.2 \mathrm{l}^{-1}$, respectively) than the SCM $\left(2.0 \mathrm{l}^{-1}\right)$. The secondary inspiratory function of the SCM, justifies its delayed recruitment. These values are representative of a mechanical advantage and were calculated by an indirect approach, based on the 
Maxwell reciprocity theorem. This theorem, when applied to the respiratory system, predicts that the potential change in the airway pressure produced by a particular muscle contracting alone against a closed airway is related to the mass of the muscle, the maximal active muscle tension per unit crosssectional area, and the fractional change in muscle length per unit volume increase of the relaxed chest wall (De Troyer et al., 1998; Legrand et al., 2003). Another variable to be considered is the mass of the muscles, because, although the action of the IC is primary, its parasternal portion weighs only $3.2 \mathrm{~g}$ (De Troyer et al., 1998). Even though the IC is active from the beginning, the small mass could explain its reduced electrical activity during higher ventilatory demand when compared to other muscles. In contrast, the masses of the SCL and SCM muscles are approximately 33.2 and $62.2 \mathrm{~g}$, respectively (Legrand et al., 2003). The fact that the SCM has almost twice as much mass as the SCL explains why they have similar muscular electrical activity despite the mechanical disadvantage of the SCM.

In summary, responding to inspiratory loads, the IC and SCL are initially recruited, but only the SCL responds by increasing its activation intensity according to the load. At loads greater than $20 \%$ of MIP, the SCM is recruited and has a similar intensity to the SCL due to its larger size. The SCM also increases its activity to match the load.

We did not find variations in the RA muscle activity in any of the comparisons made. Mesquita Montes et al. (2016) studied the effect of inspiratory and expiratory loads on the abdominal muscle activity in healthy subjects. They found results similar to ours, no variations on the RA muscle activity in response to inspiratory load, but an increased activity when using an expiratory load. Additionally, the authors described the behavior of the external oblique and transverse abdominus/internal oblique muscles. The latter reduced its activity when an inspiratory load was used, probably in order to minimize the effect of increased intra-abdominal pressure. This resulted in a greater recruitment of the diaphragm, thus revealing the contribution of the abdominal muscle during inspiration in response to increased ventilatory demand.

We did not analyze the activity of the external oblique, transverse abdominus, and internal oblique muscles. They are more sensitive to detecting abdominal muscle activity. This is a limitation to the present study. We used sEMG, which was not very effective in measuring the RA muscle activity, because of the thicker adipose layer covering. Another limitation to this study is the fact that the acute effects were assessed only for a limited period, which was necessary for the integrated analysis of the OEP and sEMG systems. However, the study presented favorable evidence on the use of inspiratory flow load

\section{REFERENCES}

Aliverti, A., Cala, S. J., Duranti, R., Ferrigno, G., Kenyon, C. M., Pedotti, A., et al. (1997). Human respiratory muscle actions and control during exercise. J. Appl. Physiol. 83, 1256-1269. doi: 10.1152/jappl.1997.83.4.1256

Aliverti, A., and Pedotti, A. (2003). Opto-electronic plethysmography. Monaldi Arch. Chest Dis. 59, 12-16. and its application to upper airways, such as a better breathing pattern and greater muscle activation. An increase in lung volume mobilization in response the imposed load favorable to causing restrictive disorders was observed, without an increase in the end-expiratory volume and therefore, allowing its use also in obstructive disorders. In addition, we believe the nasal interface could be used as an alternative for treating patients with muscle weakness or facial deformities when it is not possible to use mouthpieces. Longitudinal studies must be performed in order to identify the long-term effects of inspiratory load application on ventilation and respiratory muscle strength.

\section{CONCLUSION}

The results of our study indicate that applying inspiratory loads with a nasal interface is more effective in eliciting an increase on the inspiratory muscle activity, the chest wall and the compartmental volumes. As a perspective for future studies, we suggest evaluating the effects of IMT with a focus on the upper airways, in order to observe its repercussions on lung function.

\section{ETHICS STATEMENT}

We declare that the above mentioned manuscript was approved by the Research Ethics Committee of the Federal University of Rio Grande do Norte under protocol 1.251.451/2015, according to the Declaration of Helsinki of 1975.

\section{AUTHOR CONTRIBUTIONS}

JdF: literature search, data collection, study design, analysis of data, and manuscript preparation. KB: analysis of data and manuscript preparation. VR: review of manuscript. GF: study design and review of manuscript. AA: literature search and review of manuscript.

\section{FUNDING}

The study received financial support from the Coordenação de Aperfeiçoamento de Pessoal de Nível Superior (CAPES), PGCI CAPES 054/2014 - 23038.007514/2014-78 and Conselho Nacional de Desenvolvimento Científico e Tecnológico (CNPq) process: 400316/2012-9. GF received a grant from $\mathrm{CNPq}$ number 307353/2015-0. VR received a grant from $\mathrm{CNPq}$ number 310091/2015-2.

American Thoracic Society/European Respiratory Society (2002). ATS/ERS statement on respiratory muscle testing. Am. J. Respir. Crit. Care Med. 166, 518-624. doi: $10.1164 / \mathrm{rccm} \cdot 166.4 .518$

Araújo, P. R. S., Resqueti, V. R., Nascimento, J. Jr., Carvalho, L. D. A., Cavalcanti, A. G. L., Silva, V. C., et al. (2012). Valores de referência da pressão inspiratória nasal em indivíduos saudáveis no Brasil: estudo multicêntrico. J. Bras. Pneumol. 38, 700-707. doi: 10.1590/S1806-37132012000600004 
Barbiero, E., Vanderlei, L., Nascimento, P., Costa, M., and Scalabrini Neto, A. (2007). Influência do biofeedback respiratório associado ao padão quiet breathing sobre a função pulmonar e hábitos de respiradores bucais funcionais. Braz. J. Phys. Ther. 11, 347-353. doi: 10.1590/s1413-35552007000500004

Cala, S. J., Kenyon, C. M., Ferrigno, G., Carnevali, P., Aliverti, A., Pedotti, A., et al. (1996). Chest wall and lung volume estimation by optical reflectance motion analysis. J. Appl. Physiol. 81, 2680-2689. doi: 10.1152/jappl.1996.81.6.2680

Cunha, A. P. D. N. D., Marinho, P. É. D. M., Silva, T. N. S., França, E. É. T. D., Amorim, C., Filho, V. C. G., et al. (2005). Efeito do Alongamento sobre a Atividade dos Músculos Inspiratórios na DPOC. Saude Rev. 7, 13-19.

Da Gama, A. E., De Andrade Carvalho, L., Feitosa, L. A., Do Nascimento Junior, J. F., Da Silva, M. G., Amorim, C. F., et al. (2013). Acute effects of incremental inspiratory loads on compartmental chest wall volume and predominant activity frequency of inspiratory muscle. J. Electromyogr. Kinesiol. 23, 1269-1277. doi: 10.1016/j.jelekin.2013.07.014

De Troyer, A., Legrand, A., Gevenois, P.-A., and Wilson, T. A. (1998). Mechanical advantage of the human parasternal intercostal and triangularis sterni muscles. J. Physiol. 513, 915-925. doi: 10.1111/j.1469-7793.1998.915ba.x

Falla, D., Dall'alba, P., Rainoldi, A., Merletti, R., and Jull, G. (2002). Location of innervation zones of sternocleidomastoid and scalene muscles-a basis for clinical and research electromyography applications. Clin. Neurophysiol. 113, 57-63. doi: 10.1016/s1388-2457(01)00708-8

Han, J. N., Gayan-Ramirez, G., Dekhuijzen, R., and Decramer, M. (1993). Respiratory function of the rib cage muscles. Eur. Respir. J. 6, 722-728.

Hawkes, E. Z., Nowicky, A. V., and Mcconnell, A. K. (2007). Diaphragm and intercostal surface EMG and muscle performance after acute inspiratory muscle loading. Respir. Physiol. Neurobiol. 155, 213-219. doi: 10.1016/j.resp.2006. 06.002

Held, P. A. D. C., Silva, W. M. S., Silva, T. L. P., Di Lorenzo, K. R., and Pires, V. A. (2008). Treinamento muscular e da respiração nasal em crianças respiradoras orais. Fisioter. Mov. 21, 119-127.

Hostettler, S., Illi, S. K., Mohler, E., Aliverti, A., and Spengler, C. M. (2011). Chest wall volume changes during inspiratory loaded breathing. Respir. Physiol. Neurobiol. 175, 130-139. doi: 10.1016/j.resp.2010. 10.001

Langer, D., Charususin, N., Jácome, C., Hoffman, M., Mcconnell, A., Decramer, M., et al. (2015). Efficacy of a novel method for inspiratory muscle training in people with chronic obstructive pulmonary disease. Phys. Ther. 95, 1264-1273. doi: $10.2522 /$ ptj.20140245

Legrand, A., Schneider, E., Gevenois, P. A., and De Troyer, A. (2003). Respiratory effects of the scalene and sternomastoid muscles in humans. J. Appl. Physiol. 94, 1467-1472. doi: 10.1152/japplphysiol.00869.2002

Maarsingh, E. J., Van Eykern, L. A., Sprikkelman, A. B., Hoekstra, M. O., and Van Aalderen, W. M. (2000). Respiratory muscle activity measured with a noninvasive EMG technique: technical aspects and reproducibility. J. Appl. Physiol. 88, 1955-1961. doi: 10.1152/jappl.2000.88.6.1955

McConnell, A. K., and Romer, L. M. (2004). Respiratory muscle training in healthy humans: resolving the controversy. Int. J. Sports Med. 25, 284-293. doi: 10. 1055/s-2004-815827

Merletti, R., Farina, D., and Granata, A. (1999). Non-invasive assessment of motor unit properties with linear electrode arrays. Electroencephalogr. Clin. Neurophysiol. Suppl. 50, 293-300.

Mesquita Montes, A., Baptista, J., Crasto, C., De Melo, C. A., Santos, R., and VilasBoas, J. P. (2016). Abdominal muscle activity during breathing with and without inspiratory and expiratory loads in healthy subjects. J. Electromyogr. Kinesiol. 30, 143-150. doi: 10.1016/j.jelekin.2016.07.002
Neder, J. A., Andreoni, S., Lerario, M. C., and Nery, L. E. (1999). Reference values for lung function tests: II. Maximal respiratory pressures and voluntary ventilation. Braz. J. Med. Biol. Res. 32, 719-727. doi: 10.1590/s0100879x1999000600007

Nobre, M. E., Lopes, F., Cordeiro, L., Marinho, P. E., Silva, T. N., Amorim, C., et al. (2007). Inspiratory muscle endurance testing: pulmonary ventilation and electromyographic analysis. Respir. Physiol. Neurobiol. 155, 41-48. doi: 10. 1016/j.resp.2006.04.005

Oliveira, E. K., Silva, V. Z., and Turquetto, A. L. (2009). Relationship on walk test and pulmonary function tests with the length of hospitalization in cardiac surgery patients. Rev. Bras. Cir. Cardiovasc. 24, 478-484.

Pereira, A. C. C., Sato, T., and Carla Rodrigues, S. (2007). Novos valores de referência para espirometria forçada em brasileiros adultos de raça branca. J. Bras. Pneumol. 33, 397-406. doi: 10.1590/s1806-37132007000400008

Sanna, A., Bertoli, F., Misuri, G., Gigliotti, F., Iandelli, I., Mancini, M., et al. (1999). Chest wall kinematics and respiratory muscle action in walking healthy humans. J. Appl. Physiol. 87, 938-946. doi: 10.1152/jappl.1999.87.3.938

Sasaki, M. (2007). The effect of expiratory muscle training on pulmonary function in normal subjects. J. Phys. Ther. Sci. 19, 197-203. doi: 10.1589/jpts.19.197

Schiratzki, H. (2009). The oral and laryngeal components of the upper airway resistance during mouth breathing. Acta Otolaryngol. 60, 71-82. doi: 10.3109/ 00016486509126989

Shakouri, S. K., Salekzamani, Y., Taghizadieh, A., Sabbagh-Jadid, H., Soleymani, J., Sahebi, L., et al. (2015). Effect of respiratory rehabilitation before open cardiac surgery on respiratory function: a randomized clinical trial. J. Cardiovasc. Thorac. Res. 7, 13-17. doi: 10.15171/jcvtr. 2014.03

Soderberg, G. L., and Knutson, L. M. (2000). A guide for use and interpretation of kinesiologic electromyographic data. Phys. Ther. 80, 485-498.

Strohl, K. P., Butler, J. P., and Malhotra, A. (2012). Mechanical properties of the upper airway. Compr. Physiol. 2, 1853-1872.

Walterspacher, S., Pietsch, F., Walker, D. J., Rocker, K., and Kabitz, H. J. (2018). Activation of respiratory muscles during respiratory muscle training. Respir. Physiol. Neurobiol. 247, 126-132. doi: 10.1016/j.resp.2017. 10.004

Wilkens, H., Weingard, B., Lo Mauro, A., Schena, E., Pedotti, A., Sybrecht, G. W., et al. (2010). Breathing pattern and chest wall volumes during exercise in patients with cystic fibrosis, pulmonary fibrosis and COPD before and after lung transplantation. Thorax 65, 808-814. doi: 10.1136/thx.2009. 131409

Conflict of Interest Statement: AA is a co-inventor of the optoelectronic plethysmography whose patent rights are held by his institution, the Politecnico di Milano.

The remaining authors declare that the research was conducted in the absence of any commercial or financial relationships that could be construed as a potential conflict of interest.

Copyright (c) 2019 da Fonsêca, Resqueti, Benício, Fregonezi and Aliverti. This is an open-access article distributed under the terms of the Creative Commons Attribution License (CC BY). The use, distribution or reproduction in other forums is permitted, provided the original author(s) and the copyright owner(s) are credited and that the original publication in this journal is cited, in accordance with accepted academic practice. No use, distribution or reproduction is permitted which does not comply with these terms. 